\title{
Diffuse Reflectance Circular Dichroism for the Detection of Molecular Chirality: An Application in Remote Sensing of Flora
}

\author{
Ramon D. Wolstencroft \\ Institute for Astronomy, University of Edinburgh, EH9 $3 H J$, UK \& The \\ U.K. Astronomy Technology Centre, Royal Observatory, Edinburgh, \\ EH9 $3 H J, U . K$. \\ George E. Tranter \& Delphine D. Le Pevelen \\ Biospectroscopy Laboratory, Biological Chemistry, Biomedical Sciences \\ Division, Imperial College of Science, Technology \& Medicine, London, \\ $S W 7$ 2AZ, U.K.
}

\begin{abstract}
Terrestrial biochemistry is based upon chiral (handed) molecules, of which only one of the two possible hands is predominantly employed. This biomolecular "homochirality", may be a universal "signature" of life. Along with all living organisms, plant leaves are constructed from arrays of chiral molecules of a single hand. Through the development of a new spectroscopic approach, Diffuse Reflectance Circular Dichroism in the UV-visible wavelength regions, we have detected this chirality noninvasively from non-specular light scattered by leaves. The characteristic spectra are sensitive to plant species and leaf age/health. The technique may provide a means of remote sensing the state of flora and, at closer range, an indicator of living organisms for interplanetary landers.
\end{abstract}

\section{Introduction}

Chiral (handed) molecules are pervasive throughout terrestrial biochemistry, determining both the structure and functions of biological organisms and systems. For example, the amino acids, nucleic acids and carbohydrates, upon which all terrestrial organisms are based, are chiral. Furthermore, nature has specifically chosen predominantly to use only one of the two possible hands of these molecules: the L-amino acids and D-sugars, to the virtual exclusion of the mirror-image molecules (e.g., Fig. 1). The adoption of biomolecular homochirality enhances the efficiency of biochemistry and it is believed to be a necessary feature of all biotic chemistry - a "signature" of life (Tranter 1986). In contrast, abiotic systems have equal preference for both hands.

Plant foliage is, like all organisms, constructed of chiral molecules. In particular, the photosynthetic pigments (chromophores) are either inherently chiral (inc. chlorophyll) or are embedded in a chiral matrix of proteins, galactolipids and carbohydrates (Garrett \& Grisham 1995). Furthermore, the relative ori- 
<smiles>[R][C@@H](N)C(=O)O</smiles>

\section{L-amino acid}

Figure 1. The chirality of amino acids<smiles>[R]C(N)C(=O)O</smiles>

D-amino acid
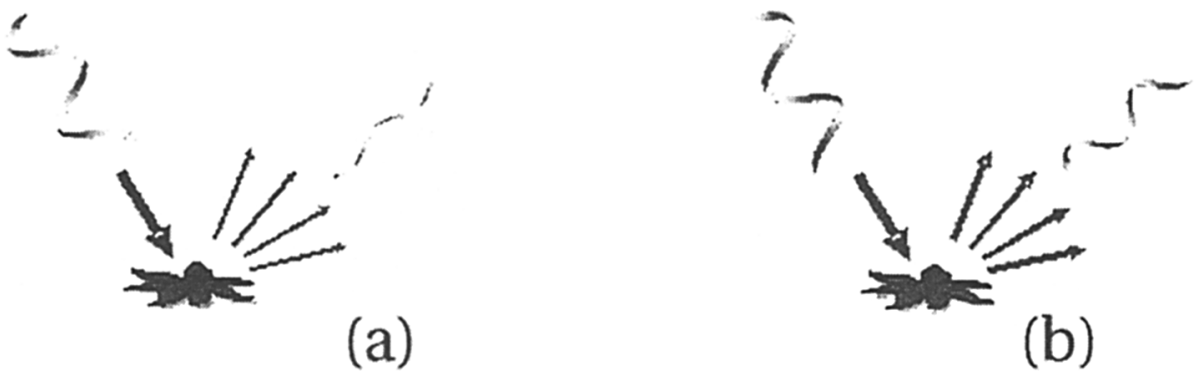

Figure 2. The differential scattering of left-handed (a) and righthanded (b) circularly polarised light from a leaf, the basis of Diffuse Reflectance Circular Dichroism.

entations of the individual chromophoric molecules with respect to each other provides an extended handed array (Glazer \& Melis 1987).

Historically, the chirality of biomolecules in solution has been probed by Circular Dichroism, a spectroscopic technique in the ultra-violet and visible wavelength region in which the differential absorption of left and right-handed circularly polarized light (Lindon et al. 2000) are measured. Here we report the results of a preliminary study using Diffuse Reflectance Circular Dichroism (DR-CD), a new technique in which the differential absorption and scattering of circularly polarized light is detected not by transmission through a solution, but by diffuse reflectance from a surface (Fig. 2).

This technique has been applied to plant foliage and is able to detect the handed environments of the leaf pigments which, in turn are indicative of the nature and state of the plant. 


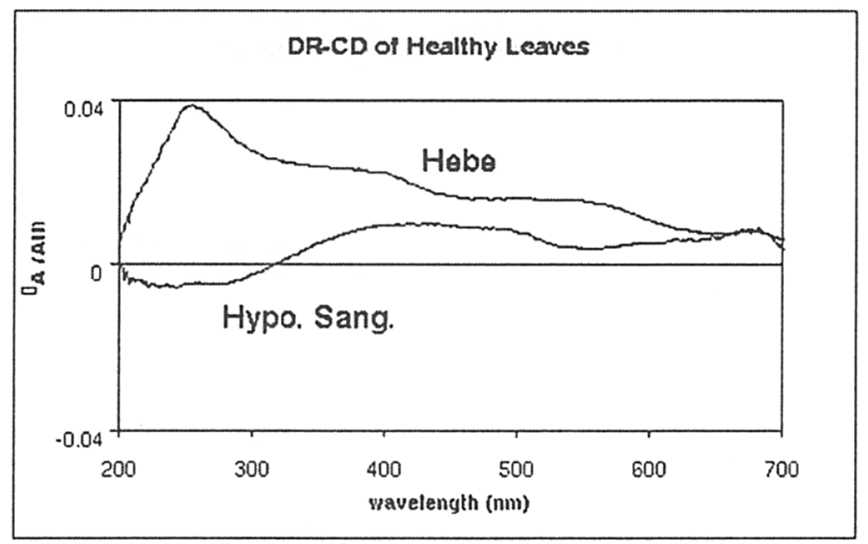

Figure 3. The DR-CD spectra of healthy leaves from Hebe and Hypoestes Sanguinolenta

\section{Materials \& Methods}

The Hebe plants were obtained from Imperial College gardens and the Hypoestes Sanguinolenta (Polka Dot Plant) from Imperial College nursery suppliers. Both were nurtured in the laboratory using the same soil, water and fertilizer.

The spectra were acquired on a Jasco J720 CD spectrometer with an adapted diffuse reflectance attachment mounted in the sample compartment. Leaves were selected and mounted in the instrument, with the upper leaf surface exposed to the incident light. The diameter of the light beam incident on the leaf was approximately $1 \mathrm{~mm}$, with the diffuse scattered light being collected by a parabolic mirror. The following instrumental parameters were employed: wave-

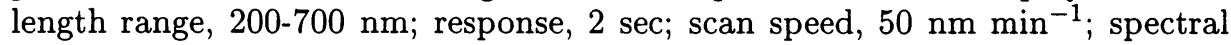
bandwidth, $5 \mathrm{~nm}$; data interval, $0.5 \mathrm{~nm}$. Spectra were background corrected by the subtraction of a corresponding DR-CD spectrum from 5 um PTFE powder acquired at a proximal time.

\section{Results \& Discussion}

The leaves of Hebe (Imperial College variety) and Hypoestes Sanguinolenta (Polka Dot Plant) are very distinctive: notably the latter has an intense pink colouration; and both types are matt in texture, ensuring that the effects of specular reflection are minimized.

The DR-CD of fresh young leaves from each plant show characteristic features. Notably, bands corresponding to the absorption by the pigment/photosynthetic chromophores are apparent (chlorophyll at $680 \& 400 \mathrm{~nm}$, carotene at $490 \mathrm{~nm}$ ). In particular, the leaves of Hypoestes Sanguinolenta have additional bands in the $550 \mathrm{~nm}$ region probably associated with phyco-based pigments.

The DR-CD response is sensitive to the state of the plant. Hebe plants were subject to various insults, including marginally dry conditions, sequential overand under-watering, and inherent chlorosis (yellowing of leaves). The DR-CD of 


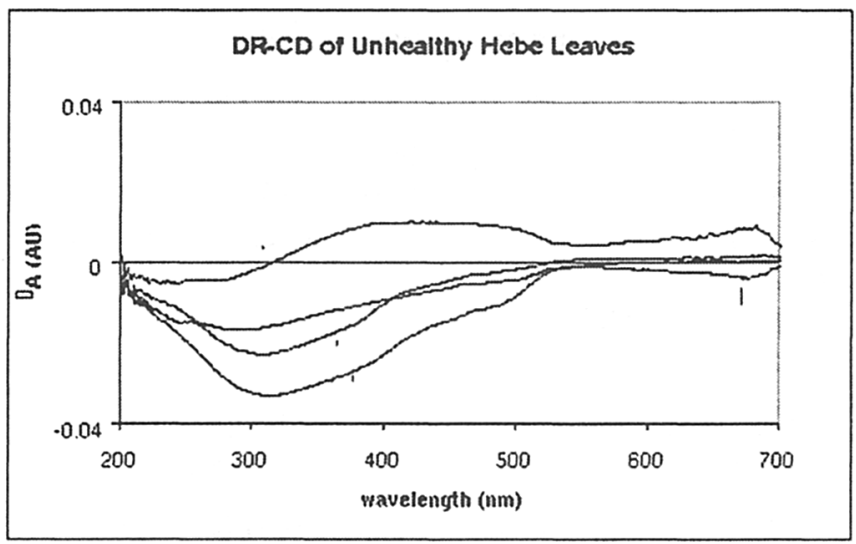

Figure 4. The DR-CD spectra of leaves from Hebe plants that were healthy (a), subject to dry conditions (b), drought-flood cycles (c) or suffering yellowing $(d)$.

representative unhealthy leaves were distinct from that of a healthy leaf. Bands at $680 \mathrm{~nm}$ were particularly reduced or lost in response to reduction or loss of photosynthetic capability, consistent with the loss of chlorophyll.

From these preliminary studies, it would seem that the DR-CD of plant foliage is indicative both of the nature of the plant and also its health. It is sensitive to the chromophores present in the leaf, their macromolecular environment, their three-dimensional alignments with each other in the photosynthetic array and the lightpath through the leaf surface layers to the pigments. Biochemical and structural differences in the leaves, whether through deterioration or from species variation, are directly manifested in the response of the leaf to the incident light (see Vogelmann 1993, for a review of plant tissue optics). In particular, the state of the chloroplasts within the mesophyll cells, containing the bulk of the chlorophyll and the other pigments, are likely to be crucial (Purves et al. 2001). Studies of further plants, under a variety of conditions, are ongoing. The detected levels of DR-CD reported here are consistent with our original studies of leaves at selected wavelengths (Wolstencroft 1974, 1996).

\section{Applications}

The existence of discernable DR-CD bands, as opposed to a continuum signal, is characteristic of specific chromophores (e.g., chlorophyll) present in the material under investigation. Thus circularly polarized scattering from, say, rough surfaces can be distinguished from true CD absorption. Furthermore, on averaging over a suitably large area, abiotic material should show no significant handedness, whereas biotic material will have a homochiral bias and thence a non-zero DR-CD.

The DR-CD results reported here were measured by illuminating the sample with alternating left and right circularly polarized light (CPL) and detecting the scattered intensity. By symmetry, the experiment could have equally been 
conducted by illuminating with unpolarised light and monitoring the intensity of scattered left and right CPL individually.

Two applications are immediately apparent:

\subsection{Remote Sensing}

The state of leaf cover on the earth's surface (rainforests, grasslands etc.) can be monitored remotely, from earth orbit or aircraft, by detecting the DR-CD using sunlight as the light source. Correspondingly, remote sensing of other planetary surfaces is a possibility.

\subsection{In Situ Analysis of Planetary Soils}

There is an opportunity for DR-CD analysis of soils and surfaces by planetary landers without the need for sampling. Direct optical measurements of scattered light can be achieved via windows or optical pipes/fibres in the lander, avoiding the huge sampling burden that is often associated with molecular analysis of planetary materials.

\section{Conclusions}

In conclusion, from the preliminary studies reported here, it is apparent that the DR-CD of leaves is characteristic of the nature of the plant and its health. The technique is non-invasive and may be applicable to remote sensing, either from close proximity or at a distance. Furthermore, it may be able to distinguish biotic from abiotic material via detection of the homochiral "signature" characteristic of biochemistry.

Acknowledgments. We would like to thank Chiralabs Ltd for their funding of this project and continued support.

\section{References}

Tranter, G. E. 1986, J. Theor. Biol., 119, 467

Garrett, R. H., \& Grisham, C. M. 1995, in Biochemistry (London: Harcourt Brace), 698

Glazer, A. N., \& Melis, A. 1987, Annual Review of Plant Physiology and Plant Molecular Biology, 38, 11

Lindon, J. C., Tranter, G. E., \& Holmes, J. L. 2000, in Encyclopedia of Spectroscopy \& Spectrometry (London: Academic Press)

Vogelmann, T. C. 1993, Annual Review Plant Physiology and Plant Molecular Biology, 44, 231

Purves, W. K., Sadava, D., Orians, G. H., \& Heller, C. 2001, in Life: The Science of Biology, 6th Ed (US: Sinauer Associates \& WH Freeman)

Wolstencroft, R. D. 1974, in Planets, Stars and Nebulae studied with photopolarimetry, ed. T. Gehrels (Tucson: University of Arizona Press), 495

Wolstencroft, R. D. 1996, in Searching for Life in the Solar System and Beyond, http://www.fas.org/spp/eprint/mars_uk/Wolstencroft.html 